Acta Hispanica (2020) Supplementum II: 829-834

\title{
ACOMPAÑAMIENTO SITUADO COMO ESTRATEGIA PEDAGÓGICA PARA EL FORTALECIMIENTO DE FORMACIÓN DOCENTE
}

\author{
Alejandra Bello Guerrero \\ Universidad de Cartagena \\ MARTHA Ligia Herrera VALdÉS \\ Secretaria de Educación Distrital de Cartagena de Indias \\ ANDres Antonio Alarcon Lora \\ Universidad de Cartagena
}

\begin{abstract}
Resumen: Este artículo está adscrito al grupo de investigación RUECA, desde la línea de investigación "Formación y Desarrollo del Profesorado" articulado con el proyecto de investigación: Estudio comparativo de formación docente de educación inicial - Región centro Perú, España y en Universidades Estatales del Caribe Colombiano, la cual da cuenta de la experiencia de formación docente desde la estrategia de acompañamiento situado que ha venido cobrando importancia dentro de la política Educativa en Colombia que propone y realiza un planteamiento integrador enfocado al mejoramiento de la práctica educativa y de los aprendizajes de los estudiantes. En Colombia se ha venido implementando esta estrategia desde diferentes ámbitos de formación docente (Maestrías en Educación, Diplomados de Formación Pedagógica, Programa Todos a Aprender, entre otros), para dar respuesta a los retos que debe enfrentar el docente de Educación Infantil como son: Atención a grupos heterogéneos, las diferencias de grupos culturales y sociales, las nuevas tecnologías etc., lo que exige del docente una formación constante que lo motive a transformar y renovar su práctica.
\end{abstract}

Palabras clave: acompañamiento situado, docencia, formación, estrategias.

\begin{abstract}
This paper is attached to the research group RUECA, from the line of research "Training and Development of Teachers" articulated with the research project: Comparative Study of Educational Training of Initial Education -Central Region 2018- Peru and Infant Education in Colombia, the which gives an account of the teacher training experience from the situated accompaniment strategy that has been gaining importance within the Educational policy in Colombia that proposes and carries out an integrating approach focused on the improvement of the educational practice and of the students' learning. In Colombia this strategy has been implemented from different areas of teacher training (Masters in Education, Diploma of pedagogical training, Program All to Learn, among others), to respond to the challenges facing the teacher of Early Childhood Education such as: attention to heterogeneous groups, the differences of cultural and social groups, new technologies, etc., which requires of the teacher a constant formation that motivates him to transform and renew his practice.
\end{abstract}

Keywords: Accompaniment Located, Teaching, Training, Strategies.

Acta Hispanica, Hungría, Supplementum II: 829-834, 2020, ISSN: 1416-7263 | 829 
Acompañamiento situado como estrategia pedagógica para el fortalecimiento de formación docente

\section{Introducción}

En Latinoamérica, incluido Colombia, los procesos dirigidos para mejorar la calidad educativa abordan diferentes escenarios, desde la adecuación en la infraestructura física de las instituciones educativas, la revisión curricular acorde a las exigencias actuales, hasta la implementación de programas pedagógicos. Sobre este último aspecto se destacan para el caso de Colombia, las iniciativas de fortalecimiento de la práctica docente a través de programas de cualificación y formación tales como: becas de maestrías, programa para la transformación de la calidad educativa "Todos a aprender" (PTA) y el Modelo de Acompañamiento Pedagógico Situado (MAS+); La características común y principal de los anteriores es el uso de la estrategia de Acompañamiento Situado, como una propuesta para mejorar los procesos en la formación docente al ofrecer un acompañamiento in situ, es decir, en el sitio donde éste desempeña su práctica, lo cual propicia el intercambio de saberes y experiencias mediante el trabajo colaborativo y cooperativo entre sus participantes.

El acompañamiento situado se constituye en un componente estratégico de formación que se centra directamente en las actividades del aula para así mejorar las prácticas docentes en su entorno y con participación de actores claves en el proceso de aprendizaje ${ }^{1}$. Su objetivo es acompañar al docente en su lugar de trabajo buscando articular y poner en práctica lo aprendido, en aras de fortalecer su proyecto de aula, haciendo énfasis en sus competencias pedagógicas, de interacción con sus compañeros y la comunidad educativa para el mejoramiento de la educación en la institución a la que pertenece.

Esta estrategia se materializa con el acompañamiento in situ del tutor quienes son "profesionales a los cuales se ha encomendado la tarea de generar condiciones para la formación, implica situar su discurso en los contextos institucionales, dialogar con ellos y generar opciones de formación para nuevos profesionales docentes" (IDEP, 2015: 19). Esta estrategia hace parte de las iniciativas diseñadas por el Estado colombiano y de las cuales se destacan tres programas para iguales espacios y cada uno indispensable para la consecución de los objetivos de política educativa, estos programas son:

\section{Programas}

\subsection{Programa Todos a Aprender (PTA)}

Formulado desde el Ministerio de Educación Nacional (MAN) para responder a la necesidad de mejorar la calidad de la educación básica primaria en Colombia y busca elevar los niveles de desempeño de los estudiantes ante la revelación, según indicadores internacionales, de promedios menores de aprendizaje y desigualdades en la calidad de la educación en el país.

\footnotetext{
${ }^{1}$ Cfr. Ministerio de Educación del Perú (2014), Ministerio de Educación Nacional de Colombia (2018).
} 
El PTA inicio en el año 2012 y se estructura en cinco componentes que articulados se convierten en las herramientas que guían la ruta de trabajo hacia la consecución de la meta propuesta; dentro de ellos se encuentra el acompañamiento de formación situada, el cual se considera como la oportunidad que los docentes tienen para mejorar su labor a partir del acompañamiento entre pares, el diálogo y la crítica.

Su desarrollo consiste en apoyar y direccionar las prácticas pedagógicas de los docentes a través de acompañamiento de un tutor al colectivo de maestros de las Instituciones Educativas focalizadas. Estos docentes de aula han sido capacitados de forma permanente en didáctica del lenguaje y matemáticas, además de recibir formación en políticas educativas.

\subsection{Modelo de Acompañamiento Pedagógico Situado (MAS+)}

Esta iniciativa se orienta específicamente al mejoramiento de la calidad en la modalidad de educación inicial, la cual es operada por el Instituto Colombiano de Bienestar Familiar (ICBF) y por las instituciones educativas oficiales del país. Su formulación, señala el MEN "responde a la necesidad del país de dar continuidad a los procesos de cualificación de las maestras de educación inicial, procurando tener un mayor un acercamiento, reconocimiento e incidencia en sus prácticas pedagógicas desde el acompañamiento presencial en el escenario educativo, y en ese mismo sentido, respondiendo con mayor contundencia a las condiciones particulares de cada uno de los entornos educativos en los que se encuentran las maestras" (s/f: 7).

\subsection{Becas excelencia}

Corresponde a una iniciativa del Gobierno colombiano para fortalecer la formación en posgrados del docente que laboran en establecimientos de educación pública inicial, básica, primaria, media y secundaria; su objetivo es mejorar la calidad de la práctica pedagógica de los establecimientos educativos, así como de las competencias básicas de los educandos en las áreas de matemáticas, lenguaje y ciencias naturales. Este programa se imparte en universidades con acreditación de alta calidad "que tengan como condición principal el desarrollo de un proyecto de fortalecimiento de la institución educativa" (Icetex, 2019).

Entre las universidades acreditadas se halla la Universidad de Cartagena con el programa Maestría en educación. La institución describe en su objetivo de enfoque pedagógico que:

Consiste en la estrategia de Acompañar al docente maestrante en su lugar de trabajo buscando articular y poner en práctica lo aprendido en la maestría, en aras de fortalecer su proyecto de investigación, haciendo énfasis en sus competencias pedagógicas, de interacción con sus compañeros y la comunidad educativa para el mejoramiento 


\section{Acompañamiento situado como estrategia pedagógica para el fortalecimiento de formación docente}

de la educación en la Institución a la que pertenece. (Universidad de Cartagena, 2015)

\section{Discusión}

Los programas descritos desde su componente muestran resultados positivos en cuanto su alcance y aplicación. A continuación se presenta las reflexiones en los programas de maestría de la universidad de Cartagena y PTA, así como los principales aspectos del informe presentado por el MEN respecto al programa MAS+.

En primer lugar se hace referencia al PTA en la ciudad de Cartagena (Bolívar) y que se caracteriza por tener diferentes enfoques que van desde lo etnoeducativo, atención a niños y niñas con dificultades auditivas, hasta población con vulneración socioeconómica.

Desde la experiencia se afirma que el acompañamiento situado reviste importancia importantes dentro del contexto educativo colombiano, en la medida que propone y realiza un planteamiento diferente e integrador enfocado hacia el acompañamiento de la práctica educativa como una estrategia de formación de docentes en ejercicio y que aporta significativamente al mejoramiento del desempeño de los maestros, además de posibilitar el desarrollo intelectual, social y técnico pedagógico de los mismos fortaleciendo su lado más humano en aras del mejoramiento de la calidad educativa y por ello se comienza a visualizar como la estrategia principal para elevar los resultados de aprendizaje de los niños y las niñas de las Instituciones Oficiales del país.

En este sentido, cabe señalar lo acotado por Montero quien sobre el acompañamiento pedagógico expresa que: "el, implica el cambiar la lógica tradicional de los sistemas masivos, homogéneos, impersonales y pasajeros propios de los grandes programas de capacitación; y llevar la formación docente al escenario y al entorno en que trabaja el maestro" (2010: 32).

Igualmente hemos participado como tutores in situ de seis investigacionessistematización de experiencias en la segunda y tercera cohorte de la maestría en educación de la Universidad de Cartagena, programa de posgrado que ha beneficiado a docentes de instituciones educativas públicas de los diferentes municipios del departamento de Bolívar.

La principal observación sobre el acompañamiento situado es sostener que esta propuesta ha facilitado la guía y apoyo de los maestrantes de las diferentes instituciones educativas beneficiadas, quienes se concientizaron que ¡no basta con la aplicación de recursos didácticos o seguir a juntillas el currículo!, es necesario hacer una reflexión de la práctica pedagógica, ajustar a su contexto, identificar las dificultades propias y externas, intercambiar los saberes derivados de la experiencia y construir o adecuar conjuntamente con los actores claves de la comunidad escolar alternativas que busquen la excelencia del proceso de aprendizaje. Adicionalmente se acota que este aprendizaje es tanto para maestrantes como para los tutores acompañantes, quienes aportan al acervo pedagógico y fortalecimiento de la estrategias situada. 
Sobre el acompañamiento situado en el MAS+, el cual se implementa cuando el tutor se encuentra con las al interior de las Unidades de Servicio y/o Instituciones Educativas se destaca en el informe presentado por el MEN ante el Congreso de la República que:

se avanzó en el posicionamiento de los protocolos que hacen parte de la guía, haciendo especial énfasis en la ruta integral de atenciones para garantizar el ingreso, la continuidad y la permanencia de los niños en el entorno educativo. Este proceso se llevó a cabo en 9 entidades territoriales (Chocó, Nuquí, Bahía Solano, Quibdó, Amazonas, Puerto Nariño, Leticia, Caldas, Anserma, Belalcázar Soledad, Tumaco) con 329 agentes educativos y docentes, con el fin de dar cierre al pilotaje de la estrategia Todos listos, que se llevó a cabo en el año 2015. Con esta estrategia, el MEN busca fortalecer las capacidades de las Secretarías de Educación, modalidades de educación inicial e instituciones educativas, en procesos clave para asegurar las transiciones en cada uno de los hitos: cambios de rutina, acogida, entrega pedagógica, entre otros (MEN, 2018: 25).

La importancia del programa se centra en la realimentación derivada de la observación directa de la práctica pedagógica; además de "las posibilidades de diálogo de saberes entre las dos partes, haciendo un reconocimiento e indagación por el conocimiento y experiencia de cada maestra, buscando construir acuerdos y objetivos personalizados para la cualificación de las prácticas" (Colombia aprende, 2012: 12).

\section{Conclusiones}

Los principales aportes del acompañamiento situado son: establecimiento de Espacios de reflexión, dinámicas de interacción a través del intercambio de saberes que fortalecen los conocimientos, nuevas formas de trabajo donde todos suman, innovación es decir adquisición de nuevas estrategias pedagógicas y actualización en cuanto a la búsqueda constante de información pertinente.

$\mathrm{El}$ acompañamiento situado se constituye en una estrategia idónea para el ejercicio de la investigación acción y la sistematización de experiencia, Adicionalmente se dimensionó el acompañamiento como una oportunidad de encuentro entre maestros dentro y fuera del aula que ha permitido recuperar el diálogo de saberes, estimulando el trabajo colaborativo.

Por último y respecto a las expectativas que tenía el programa desde sus inicios en cuanto al acompañamiento situado el PTA los docentes y el mismo establecimiento educativo asumieran como propia la dinámica de trabajo generada en los acompañamientos y que llevaran al aula las estrategias compartidas en los talleres de formación y que se consolidaran al interior de la comunidad de docentes participantes.

En el programa de maestría de la Universidad de Cartagena el acompañamiento situado se constituye en una estrategia idónea para el ejercicio de la investigación acción 
Acompañamiento situado como estrategia pedagógica para el fortalecimiento de formación docente

y la sistematización de experiencia, no obstante la verificación de resultados y diagnóstico del proceso implican una continuada sostenida a lo largo de varios años, de manera que solo se observan los resultados inmediatos de la práctica presentada en el documento de grado, pero no existe un proyecto que le dé siguiente a cómo los docentes y actores claves de la comunidad escolar asimilan, adecuan y transforman esta práctica.

\section{Referencias bibliográficas}

Anexo técnico modelo de acompañamiento pedagógico situado - MAS+. Colombia Aprende. 2012. Asequible en: http://aprende.colombiaaprende.edu.co/ckfinder/ userfiles/files/ANEXOS $\% 20$ TECNICOS $\% 20$ SUBLI $\%$ CC $\% 81$ NEAS $\% 20$ EDUCACI ON\%20INICIAL.pdf, fecha de consulta: 01-05-2019.

Becas para la excelencia docente. Icetex. 2019. Asequible en: https://www.icetex.gov.co/ dnnpro5/es-co/cr\%C3\%A9ditoeducativo/posgrado/excelenciadocente.aspx fecha de consulta: 01-05-2019.

IDEP (2015). Acompañamiento in situ como estrategia de formación docente. Bogotá: Alcaldía Mayor.

MEN (2018). Informe al Congreso de la República l 2016-2017. Bogotá: Ministerio de educación nacional de Colombia.

Ministerio de Educación (2014). Acompañamiento pedagógico. Lima: Ministerio de educación del Perú.

Universidad de Cartagena (2016) Presentación Maestría en educación. Énfasis en ciencias exactas, naturales y del lenguaje. Enfoque pedagógico: «aprendiaaje situado. Cartagena: Universidad de Cartagena. 